\title{
INFLUÊNCIA DE INFORMAÇÕES DE SAÚDE NO ESTILO DE VIDA DE PARTICIPANTES DE GINÁSTICA LABORAL
}

\author{
Influence of health information in stretch break participants' \\ lifestyle
}

\author{
Influencia de las informaciones de salud en el estilo de vida de \\ participantes de gimnasia laboral
}

Artigo Original

\begin{abstract}
RESUMO
Objetivo: Verificar a influência de informações de saúde no estilo de vida de participantes de ginástica laboral (GL). Métodos: Participaram 14 sujeitos de ambos os sexos, integrantes do programa de GL da Universidade Federal da Paraíba (UFPB), nos quais se aplicaram, entre março e maio de 2010, avaliação antropométrica e entrevista semiestruturada. A análise quantitativa utilizou média, desvio padrão e frequências absolutas. Os dados qualitativos foram baseados na análise de conteúdo, emergindo três categorias: mudanças comportamentais na prática de exercícios físicos e alimentação, lembranças de informações repassadas na avaliação antropométrica e exercícios físicos preferidos. Resultados: Os sujeitos estavam pré-obesos $\left(26,03 \mathrm{~kg} / \mathrm{m}^{2}\right)$, com risco aumentado de desenvolvimento de doenças crônicas não transmissíveis e pré-hipertensão. Cinco aumentaram a prática de exercícios físicos (EF), cinco pretendiam passar a se exercitar, três mantiveram a prática de EF, um passou a realizar EF com seu cônjuge e, dos dois que começaram a se alimentar melhor, um não pretendia se exercitar. A caminhada foi praticada por seis indivíduos. Conclusão: A transmissão de dados salutares concisos provenientes de um programa de GL pode ter reflexos positivos no estilo de vida do colaborador, sendo a caminhada o exercício físico preferido pelos participantes investigados.
\end{abstract}

Descritores: Acesso à Informação; Estilo de Vida; Saúde do Trabalhador.

\begin{abstract}
Objective: To verify the influence of health information on the lifestyle of stretch break (SB) participants. Methods: The study comprised 14 subjects of both sexes, members of the SB program of the Federal University of Paraiba (FUPB), who underwent, between March and May 2010, anthropometric evaluation and semi-structured interview. The quantitative analysis used mean, standard deviation, and absolute frequencies. The qualitative data was based on content analysis, emerging three categories: behavioural changes in the practice of physical exercise and eating, memories of information passed on the anthropometric measurements, and preferred exercise. Results: The subjects were pre-obesity $\left(26.03 \mathrm{~kg} / \mathrm{m}^{2}\right)$, with increased risk of developing chronic noncommunicable diseases and pre-hypertension. Five of them increased the practice of physical exercise (PhE), five intended to start exercising, three continued the PhE practice, one began practicing PhE with his partner and, from those two who started to eat better, one did not intend to exercise. Walking was practiced by six individuals. Conclusion: Transmission of concise health information proceeding from a SB program may have positive reflexes in the worker's lifestyle, and walking was the preferred modality of physical exercise among the investigated participants.
\end{abstract}

Clinical trials register: NCT01943734

Descriptors: Access to Information; Life Style; Occupational Health.

\author{
Raquel Suelen Brito Silva ${ }^{(1)}$ \\ Caroline Oliveira Martins ${ }^{(1)}$ \\ Leonardo Rosenstiel ${ }^{(1)}$ \\ Camilla Natasha Floriano \\ Ferreira $^{(1)}$ \\ Alexandre Sérgio Silva ${ }^{(1)}$
}

1) Universidade Federal da Paraíba - UFPB - João Pessoa (PB) - Brasil
Recebido em: 12/10/2013

Revisado em: 27/12/2013 Aceito em: 11/05/2014 


\section{RESUMEN}

Objetivo: Verificar la influencia de las informaciones de salud en el estilo de vida de participantes de gimnasia laboral (GL). Métodos: Participaron 14 sujetos de ambos sexos integrantes del programa de GL de la Universidad Federal de Paraiba (UFPB) en los cuales se aplicaron la evaluación antropométrica y entrevista semi-estructurada entre marzo y mayo de 2010. El análisis cuantitativo utilizó la media, desviación típica y frecuencias absolutas. Los datos cualitativos se basaron en el análisis de contenido emergiendo tres categorías: cambios de conducta en la práctica de ejercicios físicos y alimentares, recuerdos de informaciones repasadas en la evaluación antropométrica $y$ ejercicios físicos preferidos. Resultados: Los sujetos eran preobesos $\left(26,03 \mathrm{~kg} / \mathrm{m}^{2}\right)$, con riesgo aumentado en desarrollar enfermedades crónicas no trasmisibles y pre-hipertensión. Cinco aumentaron la práctica de ejercicios físicos (EF), cinco intentaron empezar un EF, tres mantuvieron la práctica de EF, uno empezó a realizar EF con su compañero y de los dos que empezaron a alimentarse mejor, uno no tenía la intención de practicar EF. La caminata fue practicada por seis individuos. Conclusión: La transmisión de los datos saludables concisos de un programa de GL puede reflejar positivamente en el estilo de vida del colaborador, siendo la caminata el EF elegido por los participantes investigados.

Descriptores: Acceso a la Información; Estilo de Vida; Salud Laboral.

\section{INTRODUÇÃO}

Um Programa de Promoção da Saúde do Trabalhador (PPST) encontra-se dentro de uma demanda de políticas e atividades que têm sido utilizadas no ambiente ocupacional objetivando fomentar a qualidade de vida do trabalhador (QVT) a partir do ambiente de trabalho, respeitando as características da empresa, do colaborador e da comunidade em que este se encontra ${ }^{(1)}$.

A demanda pode fazer com que um PPST seja composto, por exemplo, por palestras sobre qualidade de vida relacionada à saúde (QVRS) e ao trabalho, programa de retorno ao trabalho, programa de atendimento ao dependente químico, programa de ergonomia, informações semanais sobre QVT e programa de ginástica laboral (GL). Essa demanda é definida principalmente por meio da aplicação de questionários, checklists e avaliações biométricas. Estes e/ou outros instrumentos devem ser utilizados para avaliar se o programa ou a atividade implantada estão cumprindo seus propósitos ${ }^{(2)}$.

Assim, um questionário pode ser aplicado para identificar qual será o foco da GL em determinado setor, orientando a aplicação de exercícios de alongamento, atividades lúdicas, massagens e qualquer outra atividade que venha a ser empregada para diminuir o estresse psicofisiológico negativo, prevenir lesões, proporcionar momentos de descontração, reduzir dores no corpo, dentre vários outros benefícios ${ }^{(3-5)}$.

A coleta de dados antropométricos é decisiva para moldar e manter o PPST, mas deve ser realizada com finalidades específicas - medir por medir, sem uma finalidade específica, faz com que todos percam tempo. Os resultados da avaliação antropométrica devem ser repassados ao colaborador, principalmente para torná-lo mais responsável por melhoras em seu estilo de vida, definido ${ }^{(6)}$ como o conjunto de ações habituais que refletem as atitudes, os valores e as oportunidades na vida dos indivíduos. Assim, tal avaliação pode favorecer a aprendizagem autodirecionada e a adoção e/ou manutenção de hábitos que integram uma vida com mais qualidade ${ }^{(3,7)}$.

Apesar de avaliações antropométricas serem realizadas em programas de GL/PPST, a influência que informações provenientes dessa avaliação podem exercer sobre o estilo de vida dos avaliados não está evidenciada até o momento. A fim de preencher essa lacuna, o presente estudo teve como objetivo verificar a influência de informações de saúde no estilo de vida de participantes de GL.

\section{MÉTODOS}

Trata-se de um estudo descritivo, transversal, com abordagem quanti-qualitativa, realizado na Universidade Federal da Paraíba (UFPB).

A população-alvo foi constituída pelos 653 servidores que realizavam suas funções em 2010 na reitoria da UFPB. Durante as duas semanas iniciais da pesquisa, 43 servidores decidiram ter seus dados antropométricos coletados, sendo incluídos no presente estudo, entretanto, foram excluídos os que não completaram a avaliação antropométrica e/ou não responderam à entrevista semiestruturada, totalizando uma amostra de 14 sujeitos.

O programa de GL da UFPB teve seu piloto iniciado em outubro de 2009, em três setores da reitoria (Gabinete do Reitor, Pró-Reitoria de Assuntos Comunitários e PróReitoria de Graduação), com a aplicação de checklist $^{(2)}$ para identificar movimentos ou posturas habituais e previsíveis do trabalho que poderiam prejudicar a saúde do colaborador. Tal programa é voltado para a promoção da saúde dentro e fora do ambiente de trabalho, sendo estruturado para oferecer aulas de GL (embasadas em exercícios de alongamento) com 15 minutos de duração, atendimento semanal individualizado realizado pelo professor e disseminação semanal de informações no ambiente virtual sobre QVT e QVRS. Na etapa de identificação do programa, questionários virtuais traçaram o perfil salutar, ocupacional e de preferências do trabalhador (ex.: melhor período para 
participar das atividades do programa de GL), além de avaliação antropométrica, para individualizar ao máximo os componentes do programa de GL, viabilizando a etapa da estruturação.

A coleta dos dados ocorreu em 2010, antes do início das aulas de GL, em uma sala de reuniões no próprio ambiente de trabalho dos sujeitos, e foi realizada em dois momentos: de março a abril, realizaram-se avaliações antropométricas e em maio ocorreram as entrevistas semiestruturadas. No local citado, dispuseram-se os aparelhos de mensuração em "circuito" e foram obedecidos protocolos de coleta necessários para cada medida ${ }^{(8)}$.

$\mathrm{Na}$ avaliação antropométrica, verificaram-se as seguintes variáveis: massa corporal $(\mathrm{Kg}$, coletada por balança digital TechLine ${ }^{\circledR}$ ), estatura (cm, verificada com estadiômetro Sanny ${ }^{\circledR}$ ), perímetro da cintura (medido através do menor perímetro entre a última costela e a crista ilíaca, em $\mathrm{cm}$, mensurado por trena antropométrica flexível Sanny ${ }^{\circledR}$ ) e pressão arterial (mmHg, avaliada por esfigmomanômetro e estetoscópio Premium $\left.{ }^{\circledR}\right)$.

A partir das medidas de estatura e massa corporal, calculou-se o índice de massa corporal (IMC) em $\mathrm{kg}$ / $\mathrm{m}^{2}$, valendo-se dos pontos de corte da OMS/WHO${ }^{(9)}$, classificando como pré-obeso o indivíduo com IMC entre 25 e 29,99. Para a razão cintura-estatura (RCEst), considerou-se o resultado $\geq 0,50^{(10)}$. Para a pressão arterial - PA (mmHg) utilizaram-se pontos de corte da Sociedade Brasileira de Cardiologia ${ }^{(11)}$, indicando que a pressão arterial sistólica entre 130 e 139 ou diastólica entre 85 e 89 classifica-se como pré-hipertensão.

Uma planilha eletrônica especialmente elaborada realizou o cálculo e a classificação automática dos dados, bem como o registro do relato dos sujeitos ${ }^{(12)}$ sobre informações específicas (ex.: remédios ingeridos). A coleta de dados antropométricos de cada sujeito durou aproximadamente 30 minutos, sendo 15 minutos destinados ao repouso do indivíduo para a aferição da PA, e os 15 minutos restantes para os demais procedimentos.

Quando os trabalhadores se prontificavam para a avaliação antropométrica, respondiam a perguntas que não os habilitariam prontamente a tais medições (ter praticado previamente exercício físico - EF - ter ingerido café ou alimentos há pouco tempo, estar com a bexiga cheia). Após essa triagem, assinavam o Termo de Consentimento Livre e Esclarecido e posteriormente identificavam-se características individuais e contato (ex.: setor de trabalho, idade, e-mail). Na sequência, recebiam informações sobre os procedimentos da avaliação antropométrica e eram solicitados a permanecer em repouso por 15 minutos para a aferição da PA. Os resultados sobre o perfil salutar foram repassados aos sujeitos individualmente, de maneira que todos receberam incentivos para manter ou adotar um estilo de vida embasado na realização de atividades físicas (incluindo a prática regular de EF), além de terem sido informadas noções básicas sobre alimentação adequada (ex.: menor ingestão de alimentos gordurosos) e indicação para consultar um nutricionista quando necessário.

Em um segundo momento, realizou-se no ambiente de trabalho (em sala reservada) a entrevista semiestruturada, com duração média de dez minutos, focando na adoção ou manutenção de hábitos relacionados a EF e alimentação. Os sujeitos escolheram um codinome (nome de cidade, bairro, praia ou flor), a fim de não serem identificados por suas falas.

Para a análise dos dados quantitativos, empregou-se o SPSS $^{\circledR}$ (Statistical Package for the Social Sciences) versão 17.0, utilizando-se estatística descritiva com média e desvio padrão, bem como frequências absolutas.

Já a análise dos dados qualitativos ocorreu com base na técnica de análise de conteúdo ${ }^{(13)}$, da qual emergiram três categorias temáticas: mudanças comportamentais na prática de EF e alimentação, lembranças de informações repassadas na avaliação antropométrica e EF preferidos.Este estudo recebeu aprovação do Comitê de Ética em Pesquisa com Seres Humanos do Hospital Universitário Lauro Wanderley, da Universidade Federal da Paraíba (HULW/UFPB), sob n²98/09 em obediência a Resolução 466/12 do Conselho Nacional de Saúde.

\section{RESULTADOS E DISCUSSÃO}

Inicialmente, serão apresentados os dados quantitativos e, em seguida, os qualitativos.

Os 14 colaboradores estudados \{sexo feminino $(\mathrm{fem})=11 ; 51,07 \pm 8,17$ anos; sexo masculino $($ masc $)=3$; 51,3 anos\} desempenhavam funções técnico-administrativas na reitoria do campus I da UFPB.

Tabela I - Características antropométricas de trabalhadores da reitoria da Universidade Federal da Paraíba (UFPB) expressas em média e desvio padrão. João Pessoa-PB, 2010.

\begin{tabular}{lc}
\hline Variáveis & $\begin{array}{c}\text { Média (desvio padrão) } \\
\mathbf{n}=\mathbf{1 4}\end{array}$ \\
\hline $\mathrm{IMC}\left(\mathrm{kg} / \mathrm{m}^{2}\right)$ & $26,03( \pm 4,60)$ \\
RCEst $(\mathrm{cm})$ & $0,54( \pm 0,07)$ \\
PAS $(\mathrm{mmHg})$ & $120( \pm 13,01)$ \\
PAD $(\mathrm{mmHg})$ & $79,30( \pm 10)$ \\
\hline
\end{tabular}

IMC = Índice de massa corporal, RCEst=Razão cintura-estatura, PAS=Pressão arterial sistólica, PAD=Pressão arterial diastólica.

A Tabela I expõe as características antropométricas da amostra, indicando média de pré-obesidade, risco aumentado de desenvolvimento de DCNT e pré- 
hipertensão. Vale ressaltar que indivíduos que apresentam pré-hipertensão devem adotar um estilo de vida fisicamente ativo e saudável, sem ingestão de drogas anti-hipertensivas, sendo o tratamento indicado pela categoria mais alta da $\mathrm{PA}^{(14)}$.

A análise das falas indicou que cinco trabalhadores adotaram $(\mathrm{fem}=2 ; \quad \operatorname{masc}=1)$ ou aumentaram $\quad(\mathrm{fem}=1$; masc $=1$ ) a prática de $\mathrm{EF}$, cinco pretendiam passar a se exercitar (fem $=5)$, três mantiveram a prática de $\mathrm{EF}(\mathrm{fem}=2$; masc $=1$ ), um inclusive passou a realizar EF com seu cônjuge ( $\operatorname{masc}=1)$ e dois começaram a se alimentar melhor $(\mathrm{fem}=2)$, no entanto, dentre esses dois, um não pretendia se exercitar (fem=1).

Os EF mais praticados foram caminhada (fem $=5$; masc $=1)$, ginástica $(\mathrm{fem}=1 ; \operatorname{masc}=1)$, natação $(\mathrm{fem}=1) \mathrm{e}$ hidroginástica $(\operatorname{masc}=1)$, porém, dois indivíduos $(\mathrm{fem}=1$; masc $=1$ ) praticaram duas modalidades (caminhada $\mathrm{e}$ ginástica).

As informações repassadas na avaliação antropométrica consideradas mais importantes pelos entrevistados envolveram massa corporal $(\mathrm{fem}=4$; $\operatorname{masc}=1), \mathrm{PA}(\mathrm{fem}=2$; masc $=2)$ e perímetro da cintura $(\mathrm{fem}=3 ;$ masc $=1)$, além de indicarem que deveriam realizar mais atividades físicas $(\mathrm{fem}=3)$, que tinham risco elevado de doenças cardíacas $(\mathrm{fem}=1)$ e que apresentavam bom estado de saúde $(\operatorname{masc}=1)$.

$\mathrm{O}$ aumento significativo da prevalência das DCNT no Brasil tem resultado dos processos de transição nutricional e epidemiológica, além de serem consideradas as principais causas de mortes ${ }^{(15)}$. Dentre os fatores de risco para as DCNT, nos últimos 30 anos, tem se destacado o excesso de peso, de maneira que, para um mesmo grupo etário, as taxas de mortes são maiores entre pessoas obesas, existindo assim uma grande associação entre excesso de gordura no corpo e doenças respiratórias, cardiovasculares e articulares, por exemplo ${ }^{(16)}$.

Estudos realizados com trabalhadores brasileiros apresentaram resultados diversos, constatando-se níveis normais de IMC e $\mathrm{PA}^{(17)}$, níveis normais somente de $\mathrm{PA}^{(18)} \mathrm{e}$ alta prevalência de PA elevado e de níveis de obesidade ${ }^{(19,20)}$, principalmente entre homens. No presente estudo, a média dos resultados indicou não somente pré-hipertensão, mas também composição corporal inadequada, sugerindo a consideração de ações para a obtenção de um perfil salutar favorável. Esses dados apontam para a necessidade de medidas diversificadas na avaliação antropométrica, a fim de ser obtido um perfil salutar mais abrangente do trabalhador, ainda que sejam utilizados breves procedimentos.

As medidas do perímetro da cintura e da estatura demandam pouco tempo e oferecem dados para o cálculo da RCEst, que vêm demonstrando forte correlação com fatores de risco cardiovascular em brasileiros ${ }^{(21)}$, além de ser considerada mais sensível para a predição de riscos à saúde do que o perímetro da cintura ${ }^{(22-24)}$. Os achados da presente pesquisa envolvendo risco aumentado de desenvolvimento de DCNT também foram observados em outros estudos com trabalhadores ${ }^{(25-27)}$. A constatação de valores elevados da RCEst pode enfatizar a urgência de atividades e programas direcionados para a prevenção de DCNT, principalmente considerando que a prevenção gera menor custo quando comparada à intervenção de caráter curativo-assistencial ${ }^{(15)}$.

\section{Mudanças comportamentais na prática de exercício físicos e alimentação}

A categoria "mudanças comportamentais na prática de exercício físicos e alimentação sugere que os trabalhadores conseguiram avançar linearmente pelos estágios, relatando situações nas quais possuíam poucas informações sobre sua saúde (pré-contemplação), estavam mais conscientes de si mesmos (contemplação) e foram capazes de ter autonomia para modificar suas vidas (ação), tornando-se quem desejavam ser (manutenção). As falas a seguir exemplificam algumas situações.

"[...] voltei a caminhar e estou tentando me alimentar nos horários certos. Estou tentando comer uma comida mais sadia, como salada, que antes não tinha (na dieta)." (Jacumã)

"[...] eu aumentei (a prática de exercícios físicos), porque só fazia três vezes na semana e passei a fazer todos os dias.” (Tambaú)

“[...] também por conta de uns exames que eu fiz, que deu taxas alteradas, eu já fiz uma reeducação alimentar e foi muito proveitoso esse primeiro contato (avaliação antropométrica) com a equipe." (Flor do campo)

A fala a seguir, de Guarabira, indica que o repasse de informações após a avaliação antropométrica pode ter se caracterizado como um estímulo para que ocorressem mudanças nos hábitos relacionados a um estilo de vida fisicamente ativo e saudável por parte dos trabalhadores, capaz até de influenciar cônjuges, como evidenciado:

"[...] en e minha esposa marcamos agora caminhada, ginástica e coisas do tipo." (Guarabira)

Somente um sujeito não pretendia praticar EF (apesar de ter consciência da importância dessa prática), mas passou a se alimentar melhor, conforme demonstrou a fala de Margarida:

"Principalmente, assim... em questão da minha diabetes, através disso ai (avaliação antropométrica), eu estou melhorando um pouco minha alimentação, me cuidando mais (...). Mas eu me preparo, me preparo e nunca começo essa atividade fisica que eu sei que é essencial pro meu tratamento." (Margarida) 
A fala de Margarida evidencia que, mesmo que o trabalhador não participe de qualquer atividade física ofertada no programa de GL, ainda pode se beneficiar de outras atividades, como a avaliação antropométrica. Tal avaliação é capaz de originar melhorias em sua QVT/ QVRS, a partir do momento em que experimenta, de forma processual, um poder de decisão com base nos conhecimentos adquiridos sobre sua saúde ${ }^{(28)}$.

Mulheres possivelmente têm mais facilidade de aderir a mudanças nos hábitos de vida quando comparadas aos homens ${ }^{(29)}$, orientando que gestores de programas de GL/ PPST criem estratégias diferenciadas para promover mudança de comportamento.

Mesmo quando o perfil salutar é desfavorável (ex.: perímetro da cintura maior do que a metade da estatura do sujeito), informações sobre a condição de saúde de trabalhadores são capazes de gerar aspectos $\operatorname{positivos}^{(30)}$, demonstrando a importância da conscientização do trabalhador sobre seu real estado de saúde. Na fala de Flor do Campo, observa-se que outros procedimentos foram adotados após a avaliação antropométrica para obter uma análise aprofundada sobre sua saúde, gerando a adoção de hábitos alimentares mais saudáveis.

Isso significa que a educação em saúde é uma das soluções para conduzir pessoas a mudanças para fins de prevenção e/ou controle dos fatores de risco de DCNT por meio de hábitos saudáveis ${ }^{(30)}$. Sendo assim, uma das maneiras que podem influenciar positivamente a QVT é a participação em um programa de GL/PPST, que possibilita a adoção de comportamentos favoráveis, como ingressar em programas de condicionamento físico dentro e/ou fora do local de trabalho a partir de resultados advindos de uma simples avaliação antropométrica. A promoção de mudanças comportamentais é um fenômeno difícil de ser efetivado, mas é essencial para que os programas de GL/PPST obtenham sucesso. Considera-se o Modelo Transteórico (MT) como útil arquétipo da promoção de mudança de comportamento, possuindo basicamente cinco estágios lineares de comportamento, os quais incluem o início da mudança e a manutenção do hábito ${ }^{(31,32)}$.

\section{Lembranças de informações repassadas na avaliação antropométrica}

A categoria "lembranças de informações repassadas na avaliação antropométrica" indica que todos os sujeitos se lembravam de algo repassado na avaliação antropométrica, inclusive de informações específicas, sendo a MC (peso) a informação mais recordada. As falas de Jacumã e Guarabira (a seguir) exemplificam tais fatos.

Informações significativas podem auxiliar a motivação intrínseca, gerando um fenômeno que se caracteriza como um envolvimento em certa atividade por causa própria, por ser considerada interessante e geradora de satisfação ${ }^{(33)}$. Assim, sugere-se que informações verbais providas em avaliação antropométrica de programa de GL/PPST, além de precisas, sejam claras e facilmente assimiladas pelo trabalhador ${ }^{(2)}$, possibilitando a consolidação de um estilo de vida fisicamente ativo e saudável.

\section{"No geral, o peso, porque estava acima e propenso para a obesidade." (Jacumã) \\ "[...] minha pressão estava boa. Acredito que o peso, a circunferência, tudo isso estava nos conformes." (Guarabira)}

Vale ressaltar que tais estratégias devem ser "concretas", aliando a informação com meios para que o objetivo seja solidificado ${ }^{(2)}$. E quanto mais frequente for o estímulo aplicado em programa de GL/PPST, mudanças mais profundas e consistentes podem efetivamente ocorrer na vida dos trabalhadores ${ }^{(33)}$.

Pesquisas $^{(3,34,35)}$ verificaram como diferentes atividades ofertadas por determinado período influenciaram os hábitos de vida de trabalhadores, seja pelo aumento dos níveis de atividade física, pelo aumento da motivação ou pela vivência dos ensinamentos e repasse das informações para o meio social. Os resultados dessas pesquisas contribuem para o entendimento sobre a necessidade de estimular a participação efetiva do trabalhador em programa de GL/ PPST, a fim de que os benefícios do programa sejam significativos e duradouros. Uma das maneiras de maximizar essa participação é estimular a aderência, que se caracteriza como a manutenção da participação do trabalhador nas atividades oferecidas, considerando-se a maneira como persiste em determinado objetivo ${ }^{(2,36)}$.

Desse modo, o professor é considerado peça-chave de um programa de GL, visto que sua atuação pode ser decisiva para maximizar a aderência dos trabalhadores ao programa $^{(33)}$. O professor deve ofertar frequentes estímulos ao longo do programa de GL, inclusive apresentando a capacidade de influenciar positivamente o trabalhador que não se encontra no estágio da ação do MT. Isso requer conhecimentos conceituais e atitudinais por parte desse profissional ${ }^{(37)}$, de modo que não seja demandado grande investimento de energia física, mental e emocional para ocasionar possíveis mudanças no estilo de vida dos trabalhadores, conscientizando-os, antes de tudo, para que decidam sabiamente sobre os próprios destinos ${ }^{(38)}$.

\section{Exercícios físicos preferidos}

A categoria "exercícios físicos preferidos" demonstra que caminhar foi a atividade preferida pelos sujeitos da amostra, permitindo que adotassem ou mantivessem um estilo de vida fisicamente ativo, conforme a fala a seguir: 
"Faço caminhada de segunda a sexta, em média uma hora e 40 minutos, na praça do meu bairro." (Hortência)

A caminhada é considerada uma das atividades mais comuns entre indivíduos praticantes de $\mathrm{EF}^{(6)}$, além de contemplar os quesitos baixo investimento financeiro, flexibilidade de horário para realização e conveniência, que favorecem a prática de EF.

\section{CONSIDERAÇÕES FINAIS}

Informações verbais apresentadas em avaliação antropométrica sobre o perfil salutar de trabalhadores indicaram ter sido eficientes, principalmente porque mais da metade dos sujeitos adotou, aumentou ou manteve a prática de exercícios físicos, evidenciando que a transmissão de dados salutares concisos provenientes de um programa de GL pode ter reflexos positivos no estilo de vida do colaborador. A caminhada foi o EF preferido pelos participantes deste estudo.

Um estudo longitudinal seria fundamental para indicar a efetividade do repasse de informações em longo prazo nos diferentes comportamentos e desfechos relacionados à saúde dos trabalhadores. Sugere-se que a estratégia utilizada com a amostra do presente estudo seja enfatizada em políticas mais abrangentes, solidificando na sociedade como um todo a promoção da QVRS.

\section{AGRADECIMENTOS}

Aos graduados da Educação Física da Universidade Federal da Paraíba, ao Laboratório de Estudos e Pesquisas em Atividade Física e Saúde e ao Laboratório de Estudos do Treinamento Físico Aplicado ao Desempenho e Saúde.

Não houve conflito de interesses.

\section{REFERÊNCIAS}

1. Schimdt DRC, Paladini M, Biato C, Pais JD, Oliveira AR. Qualidade de vida no trabalho e burnout em trabalhadores de enfermagem de Unidade de Terapia. Rev Bras Enfer. 2013;66(1):130-7.

2. Martins CO. Ginástica Laboral no escritório. $2^{\mathrm{a}}$ ed. Várzea Paulista: Fontoura; 2011.

3. Ferracini GN, Valente FM. Presença de sintomas musculoesqueléticos e efeitos da ginástica laboral em funcionários do setor administrativo de um hospital público. Rev Dor. 2010;11(3):233-6.

4. Andrade PP, Veiga HMS. Avaliação dos trabalhadores acerca de um programa de qualidade de vida no trabalho: validação de escala de análise qualitativa. Psicol Ciênc Prof. 2012;32(2):304-19.
5. Couto PR, Paschoal T. Relação entre ações de qualidade de vida no trabalho e bem-estar laboral. Rev Psicol Argum. 2012;30(70):585-93.

6. Barbosa RMSP. Resenha do livro "Atividade Física, Saúde e Qualidade de Vida: conceitos e sugestões para um estilo de vida ativo", de Markus Vinicius Nahas. Rev Bras Ciênc Esporte. 2012;34(2).513-8.

7. Grande AJ, Silva J, Parra S. A. Efetividade da ginástica laboral na aptidão física estudo randomizado não controlado. Einstein (São Paulo). 2014;12(1):55-60.

8. Schneider VC, Carvalho SAT, Pereira CAM, Magalhães P. Avaliação Antropométrica em funcionários de uma penitenciária no município de Itirapina-SP. Alim Nutr Ararquara. 2011;22(4):593-9.

9. World Health Organization - WHO. Global Database of Body Mass Index - BMI classification. [acesso em 2013 Jul 2]. Disponível em: http://www.who.int/bmi/ index.jsp?introPage=intro_3.html

10. Soares DA, Barreto SM. Sobrepeso e obesidade abdominal em adultos quilombolas, Bahia, Brasil. Cad Saúde Pública. 2014;30(2): 341-354.

11. Sociedade Brasileira de Cardiologia/Sociedade Brasileira de Hipertensão/Sociedade Brasileira de Nefrologia. VI Diretrizes Brasileiras de Hipertensão. Arq Bras Cardiol. 2010;95(1 Supl 1):1-51.

12. Ferreira SRG, Moura EC, Malta DC, Sarno F. Frequência de hipertensão arterial e fatores associados: Brasil, 2006. Rev Saúde Pública. 2009;43(Supl 2):98106.

13. Gondim SMG, Bendalossi PF. Uma crítica da utilização da análise de conteúdo qualitative em psicologia. Psicol Estud. 2014;19(2):191-9.

14. Michigan Quality Improvement Consortium. Management of overweight and obesity in the adult. Southfield: Michigan Quality Improvement Consortium; 2013.

15. Malta DC, Moura EC, Castro AM, Cruz DKA, Morais Neto OL, Monteiro CA. Padrão de atividade física em adultos brasileiros: resultados de um inquérito por entrevistas telefônicas, 2006. Epidemiol Serv Saúde. 2009:18(1):7-16.

16. Azevedo ECC, Dias FMRS, Diniz AS, Cabral PC. Consumo alimentar de risco e proteção para as doenças crônicas não transmissíveis e sua associação com a gordura corporal: um estudo com funcionários da área de saúde de uma universidade. Ciênc Saúde Coletiva. 2014;19(5):1613-22. 
17. Cavalcante MA. Análise da pressão arterial domiciliar e no trabalho de auxiliares de enfermagem de um hospital de ensino. Colloquium Vitae. 2009;1(2):100-8.

18. Costa DKR, Andrade TCS, Miranda CMM, Santos CA, Peixoto HM. Prevalência de risco cardiovascular entre trabalhadores de uma instituição de ensino superior privada. Universitas Ciências Saúde. 2012;10(1):9-14.

19. Sarno F, Bandoni DH, Jaime PC. Excesso de peso e hipertensão arterial em trabalhadores de empresas beneficiadas pelo programa de alimentação do trabalhador (PAT). Rev Bras Epidemiol. 2008;11(3):453-62.

20. Ulbrich AZ, Bertin RL, Bozza R, Stabelini Neto A, Lima GZS, Carvalho T, et al. Probability of arterial hypertension from anthropometric measures in adults. Arq Bras Endocrinol Metabol. 2012;56(6): 351-7.

21. Sousa TF, Nahas MV, Silva DAS, Duca GFD, Peres MA. Fatores associados à obesidade central em adultos de Florianópolis, Santa Catarina: estudo de base populacional. Rev Bras Epidemiol. 2011;14(2):296309.

22. Bozza R, Ulbrich AZ, Lau RA, Stabelini Neto A, Mascarenhas LPG, Campos W. Associação do perímetro da cintura com o percentual de gordura e IMC em adultos de ambos os sexos de diferentes faixas etárias. Rev Bras Ativ Fís Saúde. 2012;10(2):29-36.

23. Ashwell M, Gunn P, Gibson S. Waist-to-height ratio is a better screening tool than waist circumference and BMI for adult cardiometabolic risk factors: systematic review and meta-analysis. Obes Rev. 2012;13(3):27586.

24. Haun DR, Pitanga FJG, Lessa I. Razão cintura/estatura comparado a outros indicadores antropométricos de obesidade como preditor de risco coronariano elevado. Rev Assoc Méd Bras. 2009;55(6):705-11.

25. Queiroga MR, Papini CB, Ferreira AS, Rosolem G, Kokubun E. Efeitos da idade e dos indicadores de obesidade na pressão arterial de trabalhadores. Motriz Rev Educ Fís. 2009;15(3):631-40.

26. Felipe-de-Melo ERT, Silva RCR, Assis AMO, Pinto EJ. Fatores associados à síndrome metabólica em trabalhadores administrativos de uma indústria de petróleo. Ciênc Saúde Coletiva. 2011;16(8):3443-52.

27. Berria J, Petroski EL, Minatto G. Excesso de peso, obesidade abdominal e fatores associados em servidores de uma Universidade Federal Brasileira. Rev Bras Cineantropom Desempenho Hum. 2013;15(5):535-50.
28. Wendhausen A. Educação em saúde e controle da hipertensão arterial. Alcance 2001;10(2):19-23.

29. Ferreira SRG, Moura EC, Malta DC, Sarno F. Frequência de hipertensão arterial e fatores associados: Brasil, 2006. Rev Saúde Pública. 2009;43(Supl 2):98106.

30. Spinatol IL, Monteiroll LZ, Santos ZMSA. Adesão da pessoa hipertensa ao exercício físico - uma proposta educativa em saúde. Texto \& Contexto Enferm. 2010;19(2):256-264.

31. Ribeiro AG, Cotta RMM, Ribeiro SMR. A Promoção da saúde e a prevenção integrada dos fatores de risco para doenças cardiovasculares. Ciênc Saúde Coletiva. 2012;17(1):7-17.

32. Oliveira NKR. Estágios de mudança de comportamento para a atividade física em adolescentes. Motriz Rev Educ Fís. 2012;18(1):42-54.

33. Silva RSB. Aderência de trabalhadoras em um programa de ginástica laboral ofertado em hospital público [dissertação]. João Pessoa: Universidade Federal da Paraíba; 2012.

34. Laing SS, Hannon PA, Talburt A, Kimpe S, Williams B, Harris JR. Increasing evidence-based workplace health promotion best practices in small and low-wage companies, Mason County, Washington, 2009. Prev Chronic Dis. 2012;9:110-86.

35. Candotti CT, Silva MR, Noll M, Lucchese CR. Efeito da ginástica laboral sobre a motivação para a prática regular de atividade física. Rev Baiana Saúde Pública. 2011;35(2):485-97.

36. Silva RSB, Martins CO. Otimização da aderência em programas de promoção da saúde do trabalhador. Rev Mackenzie Educ Física Esporte. 2013;12(2):228-51.

37. Kallas DB, Batista SR. A ginástica laboral como ferramenta de educação em saúde. Col Pesquisa Educação Física. 2009;8(5):7-12.

38. Grande AJ, Loch MR, Guarido EA, Costa JBY, Grande GC, Reichert FF. Comportamentos relacionados à saúde entre participantes e não participantes da ginástica laboral. Rev Bras Cineantropom Desempenho Hum. 2011;13(2):131-37.

\section{Endereço para correspondência:}

Raquel Suelen Brito Silva

Universidade Federal da Paraíba

Cidade Universitária

CEP: 58051-900 - João Pessoa - PB - Brasil

E-mail: raqueltdb4@hotmail.com 